\title{
Pattern of childbearing and mortality in married women-a national prospective study from Norway
}

\author{
Eiliv Lund, Egil Arnesen, Jens-Kristian Borgan
}

\begin{abstract}
Study objective-The aim of the study was to investigate the effect of different pattern of childbearing on total mortality.

Design-A cohort study with all currently married women aged 25 years or more at the Norwegian census in 1970 with follow up to the end of 1985 . Information on childbearing was obtained by questionnaires collected by enumerators. Follow up on death was found by a linkage based on the unique Norwegian identification number, between census information and the death register in the Central Bureau of Statistics.

Setting-The study was a national population survey.
\end{abstract}

Participants-A total of 822593 women with 11.5 million years of follow up and 112023 deaths.

Main results-Nulliparous women had higher mortality than parous women in all age groups. Parity showed a weak association with increasing mortality among high parous women. Lowest mortality was found for parous women with 2-4 children and a late first and last birth, adjusted for socioeconomic group by level of women's education.

Conclusion-The findings indicate that postponed childbearing may benefit the health of women.

Childbearing is a major biological and social event in the lives of most women, but very little is known about the relationship between the pattern of childbearing and total mortality. One large study, based on information from death certificates in England and Wales 1938-1960, ${ }^{1}$ showed that parous women had higher mortality than nulliparous, but the effect of different parity could not be studied. Another study with data from the Office of Population Censuses and Surveys found no relationship between parity and total mortality in a sample of women aged under $60 .^{2}$

The aim of this study was to analyse total mortality rates for married women in relation to different patterns of childbearing, ie, number of children (parity) and age at first and last birth.

\section{Methods}

The 1970 Norwegian census ${ }^{3}$ included all inhabitants of the country based on information from the Central Population Register of the Central Bureau of Statistics. The Central Population Register is a main source for administrative information and the key is the unique 11 digit identification number given to all persons alive at the Census in 1960, and to those born or immigrated later. The identification number consists of date of birth, sex, and control numbers. The Central Population Register is computerised and updated regularly with information on migration, immigration, emigration, and death.

The preprinted census questionnaires containing identification number, name, address, and marital status were sent to each local population registration office. The enumerators visited every household for delivery of the questionnaires. The informants had to correct any errors in the preprinted information. Shortly after the census date, 1 November 1970, the questionnaires were collected by the enumerators and returned to the Central Bureau of Statistics.

The questions on parity were only put to currently married women. ${ }^{4}$ The married women were asked about the age at marriage, the number of children in the present marriage, and the age of each child (stillborn not included). Information from the census about occupation, education, and type of employment was used by the Central Bureau of Statistics to construct a standard classification of socioeconomic status. ${ }^{56}$ In this analysis the socioeconomic status of the husband was used, divided into five broad groups; worker (unskilled or skilled), functionairy (non-manual), self employed, farmer, and pensioner. As another measure of socioeconomic status the women's own level of education was used: primary school (7 years) or higher education (8 years or more).

Follow up on mortality was obtained by a linkage based on the identification number between the files of the census and all deaths registered in the Central Bureau of Statistics for the period 1.11.1970 to 31.10.1985. Information on emigration was achieved by linking census information with emigration reports to the Central Population Register up to 31.10 .85 .

Mortality rates were based on exact personyears of follow up computed for each woman. Individuals were censored from calculation of follow up at time of emigration. Women who reimmigrated were included until death or until censored on 30.10 .85 .

The analysis was restricted to 822593 married women aged 25 years or more at the census with a total of 11533686 years of observation and 112023 deaths. For only 245 women information on either parity or age of children were missing. Direct standardisation was based on the WHO European population ${ }^{7}$ truncated to the age group 35-84 years. The number of women 85 years or older wás too small for calculation of standardised 
rates (1407 married women). Relative risk was computed according to the method of MantelHaenszel with confidence interval proposed by Miettinen. ${ }^{8}$

\section{Results}

Married nulliparous women had higher mortality rates than parous women in all age groups (table I). Nulliparous women aged 35-44 years had a relative risk ( $R R)$ of $1.60(95 \%$ confidence interval 1.48-1.74). The effect of nulliparity decreased regularly with increasing age.

Age specific total mortality after number of children showed that nulliparous women had a higher mortality than women with 2-4 children (fig 1). Women with five children or more also had a higher mortality rate than women with 2-4

Table I Age specific total mortality per 1000 years of observation, relative risk $(R R)$ with $95 \%$ confidence interval (95\% CI). Married women at the Norwegian census 1970 with follow up 1970-1985

Figure 1 Mortality per 1000 years of observation by age group and parity with directly standardised mortality rates (adjusted). Women aged 35-84 years at the Norwegian census 1970 with follow up 1970-1985

\begin{tabular}{lcc}
\hline & \multicolumn{3}{c}{ Married } \\
\cline { 2 - 3 } Age group & Nulliparous & Parous \\
\hline $23-34$ years & & \\
Rate & 0.94 & 0.79 \\
RR & 1.20 & 1.00 \\
$95 \%$ CI & $1.06-1.36$ & Reference \\
$35-44$ years & 3.32 & 2.07 \\
Rate & 1.60 & 1.00 \\
RR & $1.48-1.74$ & Reference \\
$95 \%$ CI & 7.50 & 5.07 \\
$45-54$ years & 1.48 & 1.00 \\
Rate & $1.42-1.54$ & Reference \\
RR & 16.56 & 14.01 \\
$95 \%$ CI & 1.18 & 1.00 \\
$55-64$ years & $1.15-1.22$ & Reference \\
Rate & 45.28 & 42.83 \\
RR & 1.06 & 1.00 \\
$95 \%$ CI & $1.03-1.08$ & Reference \\
$65-74$ years & 110.23 & 106.57 \\
Rate & 1.03 & 1.00 \\
RR & $0.99-1.08$ & Reference \\
95\% CI & & \\
$75-84$ years & 14.74 & 8.90 \\
Rate & 1.66 & 1.00 \\
RR & $1.63-1.68$ & Reference \\
$95 \%$ CI & & \\
$25-84$ years & & \\
Rate & &
\end{tabular}

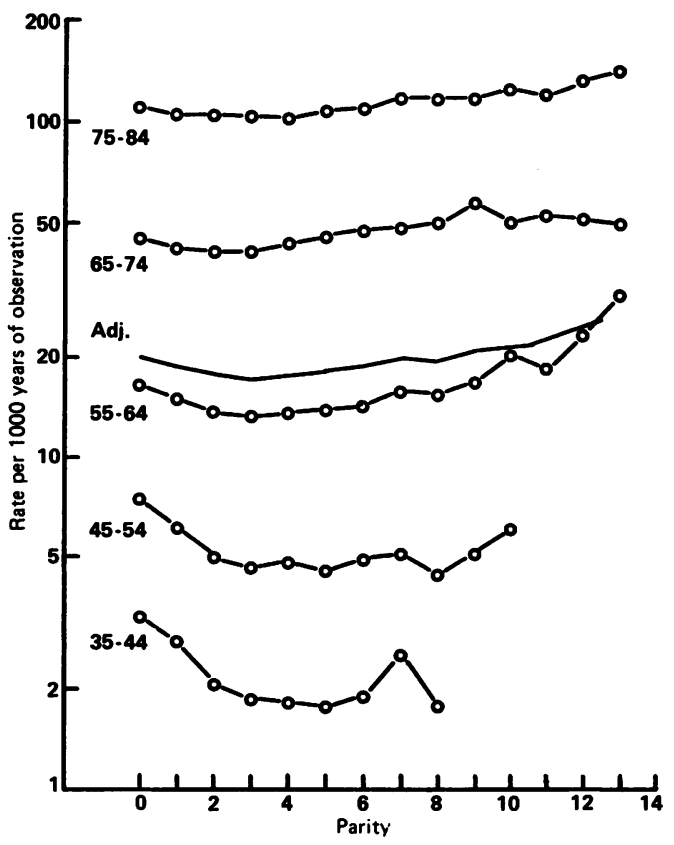

children. This effect was more pronounced in younger age groups, but can also be seen for the curve based on age adjusted mortality rate. The relative risk for nulliparous women versus women with three children was 1.39 (95\% CI 1.35-1.44), for women with six children versus women with three children 1.07 (95\% CI 1.01-1.14), and for women with 10 versus three children 1.48 (95\% CI 1.22-1.79). The relationship between parity and mortality was consistent for different age groups and for different socioeconomic groups (fig 2).

In order to look at different patterns of childbearing, an analysis stratified for parity and age at first birth was performed (table II). The lowest mortality was found for women with 3-4 children and first birth at the age of 25-29 years with relative risks of $0.65(95 \% \mathrm{CI} 0.63-0.68)$ and $0.67(95 \%$ CI $0.64-0.71)$. There were no consistent trends in all groups of parity of decreasing risks with increasing age at first birth.

The effect on mortality of education, parity, and age at first birth and at last birth showed the
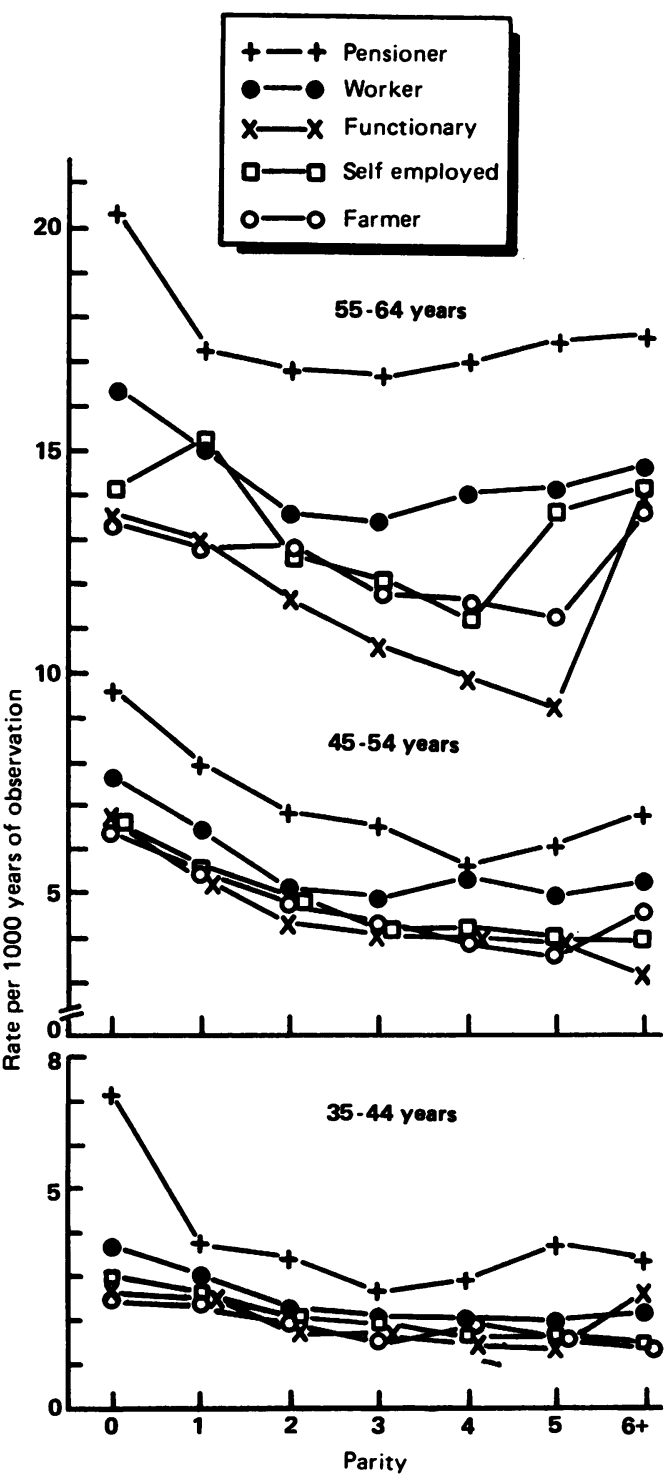

Figure 2 Mortality by parity, socioeconomic group, and age. Currently married women at the 1970 census in Norway with follow up 1970-1985 
same pattern of relative risks for both levels of education with lowest risks for women with late childbearing (table III).

\section{Discussion}

This study is the first to describe an effect on total mortality with different patterns of childbearing. Low parity and postponed births are associated with decreased total mortality.

The results are based on the observation of the total population of married women in Norway. There was no loss in the follow up, mainly as a consequence of the use of the unique identification number and population registers. Information on parity was obtained for all married women. However, some married women may have had children outside or in previous marriages. This could have been a source of misclassification bias. Analyses restricted to women who married before 35 years of age had shown that this possible misclassification bias had no effect (figures not shown). Women aged 35 years had at the census a cumulated birth rate of 2344 per 1000 women of a lifelong birth rate of 2572; those aged 40 years had a cumulative birth rate of 2437 per 1000 of a total of $2495 .^{9}$ Thus Norwegian women 35 years or older at the census had almost completely ended their fertility histories. The study can give no additional information about the effect of parity among unmarried women. The effect of factors related to other living conditions were measured either by

Table II Relative risk (RR) with 95\% confidence interval (95\% CI) according to parity and age at first birth. Married women 35-64 years at the Norwegian census 1970 with follow up 1970-1985

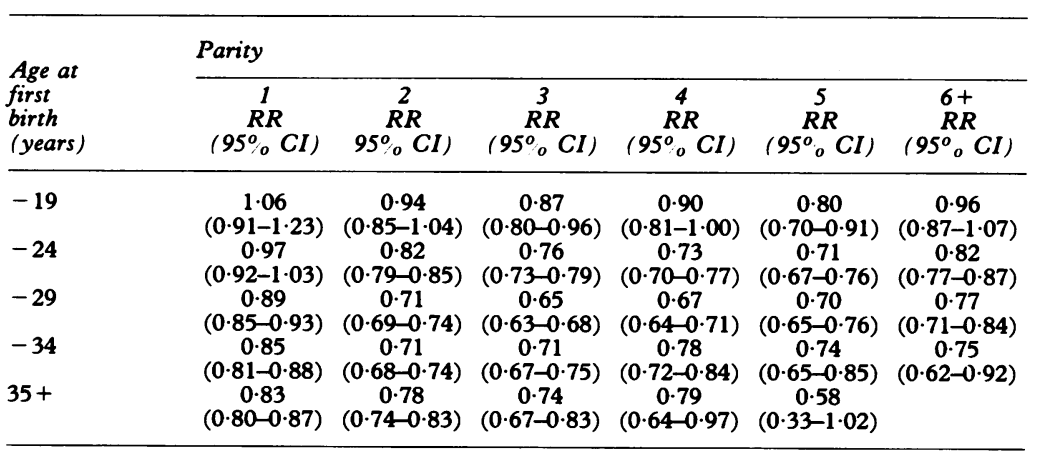

Nullipara 1.00 reference husband's socioeconomic group or by years of women's education, but neither confounded the association between pattern of childbearing and total mortality. This association between pattern of childbearing and mortality is in a highly industrialised country. Coronary heart diseases and cancer are the main causes of death.

There are few other studies on this topic. ${ }^{12}$ The only prospective study ${ }^{2}$ covered less than $1 / 10$ of the person-years of observation in this cohort. The effect of parity on total mortality was similar to the findings in this study, but the trend with increasing parity was not significant due to smaller number of deaths. However, the study had no information on age at first and last birth. Another study ${ }^{1}$ based on death certificate data from England and Wales found parous women to have a higher mortality than nulliparous, in contrast to this study. However, information on parity from a death certificate may be less valid than information given by the women herself.

The Norwegian population has experienced a major reduction in fertility from the end of the 19th century. At the census in 1920 the number of nulliparous was $9.4 \%$ versus $12.6 \%$ in 1970 , but the number of women with five children or more was $37.8 \%$ against $8.0^{\circ} 0^{4}$ Women in reproductive ages during the years between the two World Wars postponed their births. ${ }^{9}$ The changes in fertility pattern from the end of the 19th century may be part of the increased life expectancy of Norwegian women in this century. Women age 35-64 years in this cohort were born between 1905 and 1935 and thus have mainly experienced a late childbearing.

From 1945 to around 1970 a sharp increase took place in fertility rates among younger women. The reversal of fertility patterns after the Second World War could have negative effects on total mortality. In the 1980's a new strong trend in the pattern of childbearing can be observed in Norway, with first birth delayed until after the age of 25 and with an average fertility less than two children per woman. ${ }^{10}$ In 1967 , age at first birth was 23.3 years and in 1984 it increased to 25.7 years. In 1987 the average age of all mothers was 27.6 years. ${ }^{11}$ This delayed pattern of childbearing may partly be explained by more effective contraception, better and longer education among younger women, and a very high percentage of economically active women.

Table III Relative risk (RR) and $95 \%$ confidence interval (95\% CI) for death of all causes according to years of education, parity, age at first and last birth. Norwegian married women 35-64 years at the census in 1970 with follow up 1970-1985

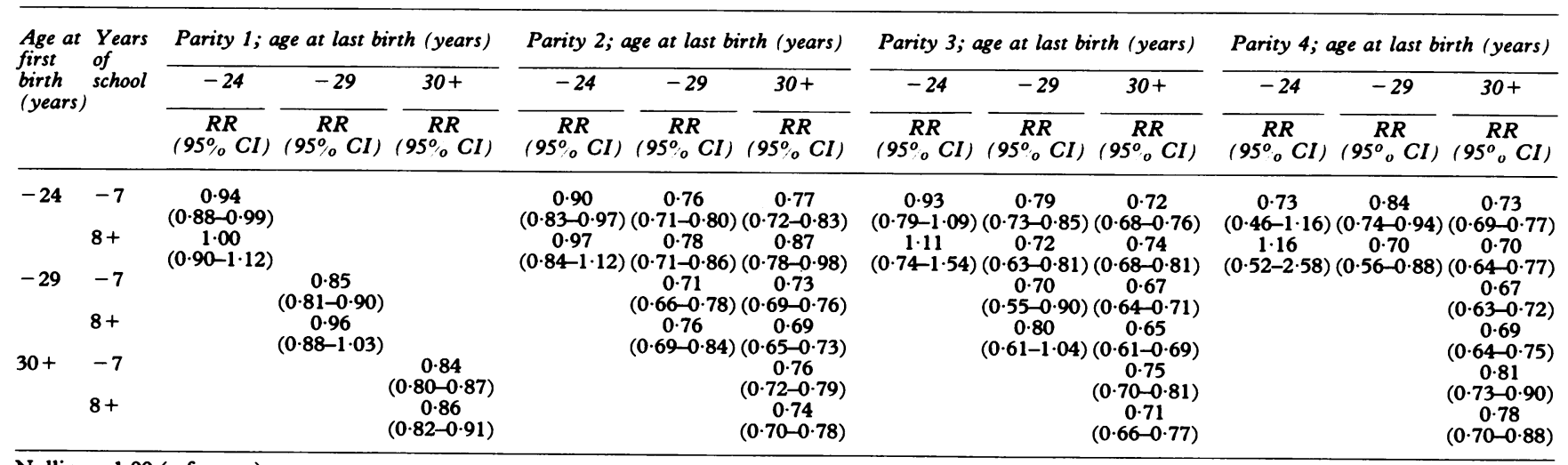


The findings in this study indicate that small family size and postponed childbearing may have beneficial effects on women's health by decreasing their mortality.

The authors thank Stefi S Wetteland for linguistic help.

1 Beral V. Long term effects of childbearing on health. $f$ Epidemiol Community Health 1985; 39: 343-6.

2 Green A, Beral V, Moser K. Mortality in women in relation to their childbearing history. Br Med $₹$ 1988; 297: 391-5. 3 Vassenden K. Folke- of boligtellingene 1960, 1970 og 1980 (The censuses 1960, 1970 and 1980). Oslo: Central Bureau (The censuses 1960, 1970 and 1980$)$
of Statistics, Report 87/2,1987.

4 Dyrvik S. Marriages and number of children-analysis of Dyrvik S. Marriages and number of children-analysis of
fertility trends in Norway 1920-1970. Oslo: Central Bureau of Statistics, Article 89, 1976.
5 Central Bureau of Statistics. Standard classification of socioeconomic status (Standarder for norsk statistikk 5). Oslo: economic status (Standarder for nor

6 Borgan J-K Kristofersen LB. Mortality by occupation and soci-economic group in Norway 1970-1980 (Statistiske soci-economic group in Norway 1970-1980 (Statistiske

7 Breslow NE, Day NE. Statistical methods of cancer research. Breslow NE, Day NE. Statistical methods of cancer research.
Volume II-The design and analysis of cohort studies. IARC Scientific Publications No. 82. Lyon: International Agency for Research on Cancer 1987.

8 Rothman KJ, Boice JD. Epidemiologic analysis with a programmable calculator. Boston: Epidemiology Resources Inc, 1982.

9 Brunborg H. Cohort and period fertility for Norway 1845 1985. Oslo: Central Burueau of Statistics, Report 88/4 1988.

10 Medical Birth Registry of Norway. Medical aspects of births, secular trends. Bergen, 1987.

11 Medical Birth registry of Norway. Annual report 1987 Bergen, 1988. 